\title{
A LITERATURE REVIEW: EFFICIENT VM MIGRATION TECHNIQUES FOR ENERGY REDUCTION IN CLOUD COMPUTING
}

\author{
Navneet Singh \\ Department of Computer Applications, \\ Sant Baba Bhag Singh University, \\ Punjab, India.
}

\author{
Dr Vijay Dhir \\ Director R \& D \\ Sant Baba Bhag Singh University, \\ Punjab, India.
}

\begin{abstract}
Broadly speaking cloud computing is nothing but a highly ‘utilitarian' orientation of IT services where users benefited on a pay-as-you go basis. In a way it enables the hosting of pervasive applications from consumer, scientific and business domain. We expect all electronic gadgetry to be 'energy efficient' to possibly achievable limits. So our data centers hosting cloud application must be cost effective and the same time should avoid undue burden of carbon footprint. While excising economy on power consumption by (data center) outmost care needs to be taken so that it never at the cost services provided to end user i.e. SLA violation must be kept as low as possible. Virtualization technology is one of the key features in cloud data centers that can improve the efficiency of hardware utilization through resource sharing, migration, and consolidation of workloads. In this paper we shall cover VM Migration Algorithms for energy reduction in cloud computing along with other novel techniques.
\end{abstract}

Keywords: Cloud Computing, Virtualization, Heuristic, Meta- Heuristic,VM migration.

\section{INTRODUCTION}

CLOUD computing has revolutionized the Information and Communication Technology (ICT) industry by enabling ondemand provisioning of elastic computing resources on a pay-as-you-go basis. Cloud computing is very much beneficial for small to medium organization. SME ( Small to medium enterprise) can save on up-font cost by outsourcing its computational needs to cloud provider and consequently costs of maintenance and upgrades. Second option is to buid private cloud within organization to boost effective resource managemnet and resource provisioning.

Today large-scale data center contains thousands of computer nodes. These data center consumes huge amount of electric power and $\mathrm{CO}_{2}$ (carbon dioxide) emission to environment. Given the increasing focus on reducing energy use, ASHRAE (American Society of Heating, Refrigerating and Air-Conditioning Engineers) recently created Standard 90.4-2016, “Energy Standard for Data Centers.”(source: http://www.achrnews.com)

Internet of things (IoT) is also a rapidly growing branch of IT sector [1]. IoT also makes us of cloud computing. Today large-scale data center contains thousands of computer nodes. These data center consumes huge amount of electric power and $\mathrm{CO}_{2}$ (carbon dioxide) emission to environment. Given the increasing focus on reducing energy use, ASHRAE (American Society of Heating, Refrigerating and Air-Conditioning Engineers) recently created Standard 90.4-2016, “Energy Standard for Data Centers.”(source: http://www.achrnews.com).

Eucalyptus cloud software is widely used for creating private cloud for organization[2].

\section{NEED OF STUDY}

The only drawback with cloud computing is that it is a notorious power guzzlers and call for a stringent 'energy efficiency' regime[3]. Currently it is estimated that servers consume $0.5 \%$ of the world's total electricity usage. Server energy demand doubles every 4-6 years. With their enormous appetite for energy, today's data centers emit as much carbon dioxide as all of Argentina. Data center emissions are expected to quadruple by 2020. The average data center consumes as much energy as 25,000 households reported by Kaplan et al[4].

Between 2000 and 2007, the total power consumption of datacenters worldwide went from 70 billion to 330 billion $\mathrm{kWh}$; it's projected to grow to more than 1,000 billion kWh by 2020. In 2003, the power density of a single rack of servers was between $250 \mathrm{~W}$ and $1.5 \mathrm{~kW}$. In 2014, it had reached almost $10 \mathrm{~kW}$ and is projected to reach up to $30 \mathrm{~kW}$ by 2020 .

"In the actual scenario, with an average Power Usage Efficiency (PUE) of 1.8, worldwide data center energy consumption will reach 507.9 TWh by 2020", explains Mattin Grao Txapartegy, Technology \& Market Analyst at Yole.”

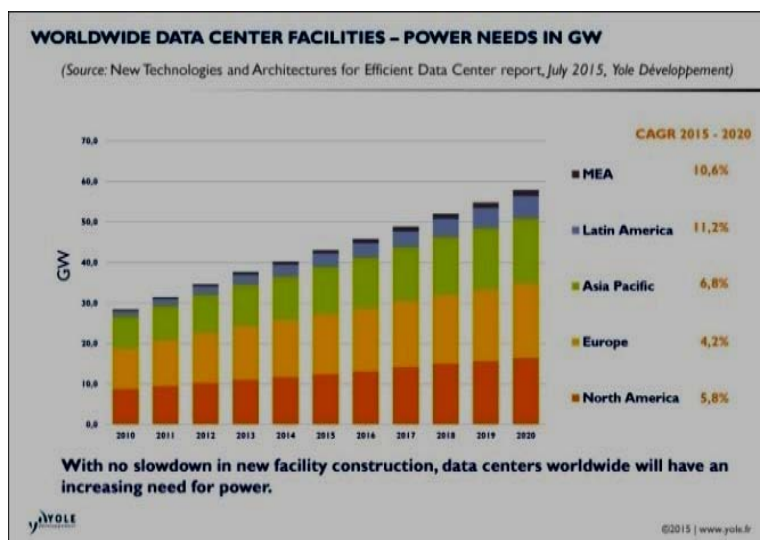

Source : http://www.yole.fr 


\section{VIRTUAL MACHINE MIGRATION TECHNIQUES}

The process of movement of virtual machine instances from one physical node or storage location to another is called VM migration. We are living in an age, where something may be manipulated or altered with the assistance of advance technology [5].

\subsection{Live Migration and High Availability}

Live migration is movement of a virtual machine instance from one physical host to anther while being powered on. Live migration is helpful in load balancing or while performing proactive maintenance in case of failure of physical machine.

\subsection{Clod/Regular Migration}

Cold migration can be defined as migration of powered-off or suspended virtual machine. Cold migration is simple to implement as compared to live migration.

By using cold migration you can also move associated disks from one data store to another.

Before you perform VM migration to reduce energy consumption of a data center following points must be analyzed:

(a)When to migrate VMs[6] Determine the best time to migrate the VM instance to reduce energy consumption without violating SLA.

(b)Which VMs to migrate: once decision has been taken to perform VM migration, next step is select set VM instance for migration from one host to another; that results in effective usage of resources in cloud.

(c) Where to migrate the VMs selected for migration[6]: select the set of physical machines on which VM instances to be migrated.

d)When and which physical nodes to switch on/off: Last step the put the idle nodes to power saver mode or hibernation to reduce energyconsumption.

\section{CLASSIFICATION OF VM MIGRATION ALGORITHMS FOR ENERGY REDUCTION IN CLOUD COMPUTING}

Since efficient VM allocation \& migration helps in energy reduction of data center, which is often modeled as bin packing problem and has been proved as NP-hard problem [7]. While excising economy on power consumption by (data center) outmost care needs to be taken so that it never at the cost services provided to end user i.e. SLA violation must be kept as low as possible. Fig 1 shows Classification of VM migration Algorithms.

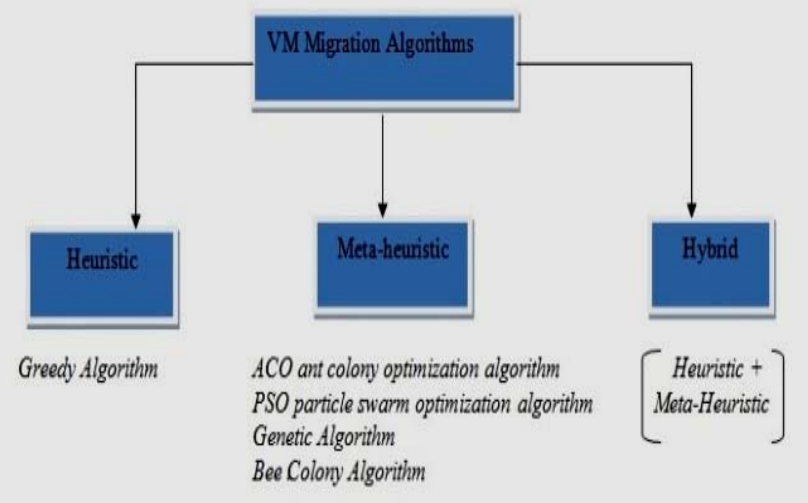

Fig 4 Classification of VM migration Algorithms.

\subsection{HEURISTIC ALGORITHMS}

Heuristic is a set of constraints that aim at finding a good solution for a particular problem [8]. The set of constraints used by heuristic are problem dependent and provide solution to a problem in a limitde time. These heuristic methods have various constraints like number of migrations, SLA, cost, etc. There is need to construct optimization functions in different ways. The main plus point of heuristic algorithms is that they give satisfactory solution to a problem in limited time cost frame. Heuristic algorithms are easier to implement in comparison to meta-heuristic algorithms. Since heuristic algorithms run faster, they are more suitable for online task scheduling that requires minimum response time. Greedy algorithm is a type of heuristic algorithms and is applied in the literature [9][10][11] to quickly obtain a solution for online scheduling scenario.

\subsection{META-HEURISTIC ALGORITHMS}

Meta -heuristic algorithms are mainly designed for a general purpose problem. They follow uniform set of procedures to construct and solve problems. The typical meta-heuristic algorithms are bio-inspired like genetic algorithms, ACO (Ant colony optimization ,Particle Swarm Optimization and honey bee foraging algorithms.

\subsection{HYBRID ALGORITHMS}

In Hybrid algorithms, heuristic algorithms are used to provide initial VM placement and meta-heuristic algorithms provides optimum placement of VMs during migration. This algorithm increases the implementation complexity but reduces time and cost space. Thiruvenkadam at el[12] proposed a hybrid genetic algorithm that follows this approach. 
5. SUMMARY CONSTRAINT

\begin{tabular}{|c|c|c|c|c|c|c|}
\hline Scheme( Ref.) & Description & $\begin{array}{l}\text { Experimental } \\
\text { Configuration }\end{array}$ & $\begin{array}{l}\text { Technique } \\
\text { used }\end{array}$ & workload & Evaluation & $\begin{array}{l}\text { Performance } \\
\text { improvement }\end{array}$ \\
\hline $\begin{array}{l}\text { VRDI (WEN } \\
\text { et al.[13]) }\end{array}$ & $\begin{array}{l}\text { Virtual resource } \\
\text { dynamic } \\
\text { integration } \\
\text { (VRDI) method } \\
\text { is based on the } \\
\text { live migration } \\
\text { technology of } \\
\text { VM, which can } \\
\text { reduce the } \\
\text { energy } \\
\text { consumption of a } \\
\text { data center by } \\
\text { integrating the } \\
\text { virtual resources. }\end{array}$ & $\begin{array}{l}\text { Simulation } \\
\text { with } 100 \text { PMs. }\end{array}$ & $\begin{array}{l}\text { Used the } \\
\text { encoding, } \\
\text { crossover and } \\
\text { mutation } \\
\text { operations of } \\
\text { the genetic } \\
\text { algorithm }\end{array}$ & $\begin{array}{l}\text { Synthetic } \\
\text { data }\end{array}$ & $\begin{array}{l}\text { Simulation } \\
\text { on } \\
\text { CloudSim } \\
\text { using } \\
\text { synthetic } \\
\text { data }\end{array}$ & $\begin{array}{l}\text { i) The VRDI } \\
\text { method can } \\
\text { save about } \\
45 \% \text { of } \\
\text { energy when } \\
\text { the resource } \\
\text { utilization of } \\
\text { PM is less } \\
\text { than 50\%. } \\
\text { (ii)SLA } \\
\text { violation of } \\
\text { the VRDI } \\
\text { method is } \\
\text { lower than } \\
\text { that of the } \\
\text { BMH and } \\
\text { ACS-VMC } \\
\text { methods. (iii) } \\
\text { the number of } \\
\text { VMs to be } \\
\text { migrated in } \\
\text { the VRDI } \\
\text { method is } \\
\text { larger than the } \\
\text { BMH and } \\
\text { ACS-VMC } \\
\text { methods. }\end{array}$ \\
\hline $\begin{array}{l}\text { ECR (WANG } \\
\text { et al. [14]) }\end{array}$ & $\begin{array}{l}\text { New task model } \\
\text { that describes the } \\
\text { QoS } \\
\text { requirements of } \\
\text { tasks with the } \\
\text { minimum } \\
\text { frequency. Used } \\
\text { energy } \\
\text { consumption } \\
\text { ratio (ECR) to } \\
\text { evaluate the } \\
\text { efficiency of } \\
\text { different } \\
\text { frequencies } \\
\text { under which to } \\
\text { execute a take. }\end{array}$ & $\begin{array}{lr}\text { three } & \text { Dell } \\
\text { R720 servers } \\
\text { with CentOS }\end{array}$ & DVFS & $\begin{array}{l}\text { use NAS } \\
\text { Parallel } \\
\text { Benchmar } \\
\text { ks } 3.3 \\
\text { (NPB) to } \\
\text { generate } \\
\text { the testing } \\
\text { tasks }\end{array}$ & $\begin{array}{l}\text { real test-bed } \\
\text { system and } \\
\text { simulation }\end{array}$ & $\begin{array}{l}\text { i) OECR } \\
\text { (energy-aware } \\
\text { method } \\
\text { Optimal } \\
\text { Energy } \\
\text { Consumption } \\
\text { Ratio) can } \\
\text { save more } \\
\text { than } 15 \% \\
\text { energy as } \\
\text { compared to } \\
\text { FFD } \\
\text { algorithm. }\end{array}$ \\
\hline
\end{tabular}




\begin{tabular}{|c|c|c|c|c|c|c|}
\hline Scheme( Ref.) & Description & $\begin{array}{l}\text { Experimental } \\
\text { Configuration }\end{array}$ & $\begin{array}{l}\text { Technique } \\
\text { used }\end{array}$ & workload & Evaluation & $\begin{array}{l}\text { Performance } \\
\text { improvement }\end{array}$ \\
\hline $\begin{array}{l}\text { Self-organized } \\
\text { criticality } \\
\text { Approach } \\
\text { (Laredo et al. } \\
\text { [15]) }\end{array}$ & $\begin{array}{l}\text { Self-organized } \\
\text { criticality } \\
\text { approach for } \\
\text { dynamically } \\
\text { load-balancing } \\
\text { computational } \\
\text { workloads. This } \\
\text { approach is able } \\
\text { to converge } \\
\text { towards near- } \\
\text { optimal trade- } \\
\text { offs where the } \\
\text { energetic } \\
\text { efficiency and } \\
\text { the QoS are } \\
\text { maximized. }\end{array}$ & $\begin{array}{l}\text { Simulation } \\
\text { with } 100 \text { nodes }\end{array}$ & $\begin{array}{l}\text { Bak-Tang- } \\
\text { Wiesenfeld } \\
\text { sandpile }\end{array}$ & $\begin{array}{l}\text { real trace } \\
\text { from the } \\
\text { Grid } \\
\text { Workload } \\
\text { s Archive }\end{array}$ & $\begin{array}{l}\text { Simulation } \\
\text { on sandpile } \\
\text { simulator } \\
\text { and real } \\
\text { traces. }\end{array}$ & $\begin{array}{l}\text { Minimizes } \\
\text { the energy } \\
\text { consumption } \\
\text { (i.e., number } \\
\text { of active } \\
\text { resources } \\
\text { through time) } \\
\text { and } \\
\text { maximizing } \\
\text { the QoS (i.e., } \\
\text { the average } \\
\text { waiting time } \\
\text { of tasks in the } \\
\text { system }\end{array}$ \\
\hline $\begin{array}{l}\text { Dynamic } \\
\text { overbooking } \\
\text { strategy (Son } \\
\text { et al. [16]) }\end{array}$ & $\begin{array}{l}\text { By using } \\
\text { dynamic } \\
\text { overbooking } \\
\text { strategy, they } \\
\text { proposed } \\
\text { dynamic } \\
\text { overbooking } \\
\text { algorithms } \\
\text { (ConnCons } \\
\text { +MostCorr and } \\
\text { ConnDist+Least } \\
\text { Corr). }\end{array}$ & $\begin{array}{l}\text { Simulation } \\
\text { with } 128 \text { hosts }\end{array}$ & $\begin{array}{l}\text { dynamic } \\
\text { overbooking } \\
\text { strategy }\end{array}$ & $\begin{array}{l}\text { Wikipedia } \\
\text { workloads }\end{array}$ & $\begin{array}{l}\text { Simulation } \\
\text { on } \\
\text { CloudSimS } \\
\text { DN }\end{array}$ & $\begin{array}{l}\text { Performs } \\
\text { better as } \\
\text { compared to } \\
\text { baseline } \\
\text { algorithms } \\
\text { NoOver and } \\
\text { ConnNone) in } \\
\text { terms of } \\
\text { energy } \\
\text { consumption } \\
\text { and SLA } \\
\text { violation. }\end{array}$ \\
\hline $\begin{array}{l}\text { NSGA-II } \\
\text { (ZHENG et al. } \\
[17])\end{array}$ & $\begin{array}{l}\text { Non-dominated } \\
\text { sorting genetic } \\
\text { algorithm } \\
\text { (NSGA-II) and a } \\
\text { local selection } \\
\text { strategy based on } \\
\text { fuzzy is used to } \\
\text { generate a } \\
\text { Hybrid Energy- } \\
\text { Aware Resource } \\
\text { Allocation } \\
\text { Approach in } \\
\text { Cloud } \\
\text { Manufacturing } \\
\text { Environment }\end{array}$ & $\begin{array}{l}\text { dual } 2.63 \mathrm{GHz} \\
\text { Intel } \\
\text { processor and } \\
\text { 8 GB RAM. }\end{array}$ & $\begin{array}{l}\text { Genetic \& } \\
\text { fuzzy }\end{array}$ & $\begin{array}{l}\text { Data } \\
\text { collected } \\
\text { from } 300 \\
\text { high- } \\
\text { speed } \\
\text { train } \\
\text { manufactu } \\
\text { rers. }\end{array}$ & $\begin{array}{l}\text { Simulation } \\
\text { on Matlab } \\
\text { 2010b }\end{array}$ & $\begin{array}{l}\text { Minimizes the } \\
\text { energy } \\
\text { consumption } \\
\text { as compared } \\
\text { to base line } \\
\text { algorithm. }\end{array}$ \\
\hline $\begin{array}{l}\text { Multiagent } \\
\text { (MA)-based } \\
\text { VM allocation } \\
\text { approach } \\
\text { (Wang et al. } \\
{[18] \text { ) }}\end{array}$ & $\begin{array}{l}\text { MA works by } \\
\text { first dispatching } \\
\text { a cooperative } \\
\text { agent to each PM } \\
\text { to assist the PM } \\
\text { in managing VM } \\
\text { resources } \\
\text { efficiently. }\end{array}$ & $\begin{array}{l}\text { Each cloud } \\
\text { system } \\
\text { consists of } 128 \\
\text { PMs.( Tree } \\
\text { Network, } \\
\text { BCube } \\
\text { Network, } \\
\text { Lattice-Like } \\
\text { Network) }\end{array}$ & $\begin{array}{l}\text { distributed } \\
\text { artificial } \\
\text { intelligence }\end{array}$ & $\begin{array}{l}\text { Synthetic } \\
\text { data }\end{array}$ & Simulation & $\begin{array}{l}\text { Compared } \\
\text { with } \\
\text { traditional } \\
\text { centralized } \\
\text { bin packing- } \\
\text { based BFD } \\
\text { approach, MA } \\
\text { performs } \\
\text { better in terms } \\
\text { of energy \& } \\
\text { migration } \\
\text { cost. }\end{array}$ \\
\hline
\end{tabular}




\begin{tabular}{|c|c|c|c|c|c|c|}
\hline Scheme( Ref.) & Description & $\begin{array}{l}\text { Experimental } \\
\text { Configuration }\end{array}$ & $\begin{array}{l}\text { Technique } \\
\text { used }\end{array}$ & workload & Evaluation & $\begin{array}{l}\text { Performance } \\
\text { improvement }\end{array}$ \\
\hline $\begin{array}{l}\text { Novel } \\
\text { approach for } \\
\text { long-term } \\
\text { predictions of } \\
\text { resource } \\
\text { demands of } \\
\text { virtual } \\
\text { machines for } \\
\text { host overload } \\
\text { detection. } \\
\text { (Minarolli et } \\
\text { al. [19]) }\end{array}$ & 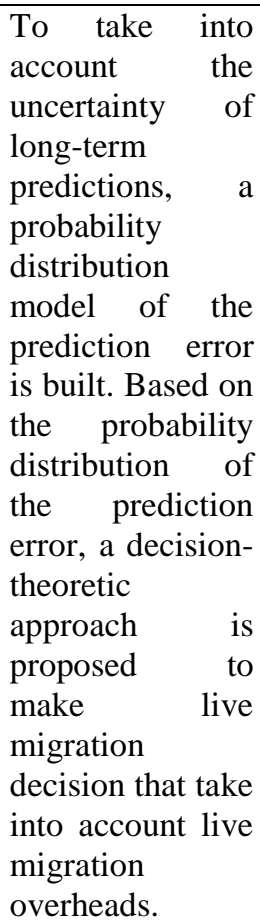 & $\begin{array}{l}\text { Simulation } \\
\text { with } 100 \\
\text { heterogeneous } \\
\text { hosts. }\end{array}$ & $\begin{array}{ll}\text { machine } & \\
\text { learning } & \\
\text { approach } & \\
\text { based } & \text { on } \\
\text { Gaussian } & \\
\text { Processes } & \end{array}$ & $\begin{array}{l}\text { PlanetLab } \\
\text { workloads }\end{array}$ & $\begin{array}{l}\text { CloudSim } \\
\text { simulator }\end{array}$ & $\begin{array}{l}\text { Achieves } \\
\text { better } \\
\text { performance } \\
\text { and higher } \\
\text { stability } \\
\text { compared to } \\
\text { other } \\
\text { approaches. }\end{array}$ \\
\hline $\begin{array}{l}\text { MOACS } \\
\text { (Ashraf et al. } \\
[20])\end{array}$ & $\begin{array}{l}\text { Multi-objective } \\
\text { ant colony } \\
\text { system } \\
\text { [MOACS] } \\
\text { algorithm for } \\
\text { virtual machine } \\
\text { consolidation in } \\
\text { cloud data } \\
\text { centers. }\end{array}$ & $\begin{array}{l}\text { Intel Core i7- } \\
4790 \\
\text { Processor with } \\
16 \text { gigabytes } \\
\text { of memory. }\end{array}$ & $\begin{array}{l}\text { multi- } \\
\text { objective ant } \\
\text { colony } \\
\text { system }\end{array}$ & $\begin{array}{l}\text { Synthetic } \\
\text { data }\end{array}$ & $\begin{array}{l}\text { Simulation } \\
\text { using Java } \\
\text { program }\end{array}$ & $\begin{array}{l}\text { maximize the } \\
\text { number of } \\
\text { released PMs } \\
\text { thus reduces } \\
\text { energy } \\
\text { consumption } \\
\text { as compared } \\
\text { to two } \\
\text { existing ant } \\
\text { colony } \\
\text { optimization } \\
\text { based VM } \\
\text { consolidation } \\
\text { algorithms } \\
\text { [Feller-ACO } \\
\text { algorithm \& } \\
\text { single- } \\
\text { objective, } \\
\text { single-colony } \\
\text { ACS VM } \\
\text { consolidation } \\
\text { algorithm]. }\end{array}$ \\
\hline $\begin{array}{l}\text { Just-in-time } \\
\text { adaptive } \\
\text { workflow } \\
\text { scheduling } \\
\text { heuristics } \\
\text { (POOLA et al. } \\
\text { [21]) }\end{array}$ & $\begin{array}{l}\text { Just-in-time } \\
\text { adaptive } \\
\text { workflow } \\
\text { scheduling } \\
\text { heuristics uses } \\
\text { on-demand and } \\
\text { spot instances to } \\
\text { provide fault- } \\
\text { tolerant } \\
\text { schedules whilst } \\
\text { minimizing time } \\
\text { and cost. }\end{array}$ & $\begin{array}{l}\text { VMs/cloud } \\
\text { resources are } \\
\text { modeled } \\
\text { similar to } \\
\text { Amazon EC2 } \\
\text { instances. }\end{array}$ & $\begin{array}{l}\text { ECPTR(Esse } \\
\text { ntial Critical } \\
\text { Path Task } \\
\text { Replication) } \\
\text { and } \\
\text { CTR(Critical } \\
\text { Task } \\
\text { Replication) }\end{array}$ & $\begin{array}{l}\text { Amazon } \\
\text { spot } \\
\text { market } \\
\text { traces }\end{array}$ & CloudSim & $\begin{array}{l}\text { Cost of } \\
\text { ECPTR is } \\
\text { much lower } \\
\text { than the base } \\
\text { line } \\
\text { ECPTRRM } \\
\text { (Essential } \\
\text { Critical Path } \\
\text { Task } \\
\text { Replication } \\
\text { without } \\
\text { Resource } \\
\text { Maximization } \\
\text { ) algorithm. }\end{array}$ \\
\hline
\end{tabular}




\begin{tabular}{|c|c|c|c|c|c|c|}
\hline Scheme( Ref.) & Description & $\begin{array}{l}\text { Experimental } \\
\text { Configuration }\end{array}$ & $\begin{array}{l}\text { Technique } \\
\text { used }\end{array}$ & workload & Evaluation & $\begin{array}{l}\text { Performance } \\
\text { improvement }\end{array}$ \\
\hline $\begin{array}{l}\text { VMCUP-M } \\
\text { (Hieu et al. } \\
[22])\end{array}$ & $\begin{array}{l}\text { virtual machine } \\
\text { consolidation } \\
\text { algorithm with } \\
\text { multiple usage } \\
\text { prediction } \\
\text { (VMCUP-M) to } \\
\text { improve the } \\
\text { energy efficiency } \\
\text { of cloud data } \\
\text { centers }\end{array}$ & $\begin{array}{l}\text { Simulation } \\
\text { with } 800 \\
\text { heterogeneous } \\
\text { hosts }\end{array}$ & $\begin{array}{l}\text { VM } \\
\text { consolidation }\end{array}$ & $\begin{array}{l}\text { Real } \\
\text { workload } \\
\text { traces in } \\
\text { the } \\
\text { Google } \\
\text { Cluster } \\
\text { Data } \\
\text { dataset \& } \\
\text { PlanetLab. }\end{array}$ & $\begin{array}{l}\text { CloudSim } \\
\text { simulation } \\
\text { toolkit }\end{array}$ & $\begin{array}{l}\text { VMCUP-M } \\
\text { reduces } \\
\text { energy } \\
\text { consumption } \\
\text { compared to } \\
\text { the multiple } \\
\text { resource } \\
\text { black-box and } \\
\text { graybox (BG) } \\
\text { scheme. }\end{array}$ \\
\hline $\begin{array}{l}\text { HCLB } \\
\text { (Pantazoglou } \\
\text { et al. [23]) }\end{array}$ & $\begin{array}{l}\text { HyperCube Load } \\
\text { Balancer(HCLB) } \\
\text { is a decentralized } \\
\text { approach } \\
\text { towards scalable } \\
\text { and energy- } \\
\text { efficient } \\
\text { management of } \\
\text { virtual machine } \\
\text { (VM) instances. }\end{array}$ & \begin{tabular}{l}
\multicolumn{2}{l}{ Simulation } \\
with $\quad 1,024$ \\
compute nodes
\end{tabular} & $\begin{array}{l}\text { VM } \\
\text { consolidation }\end{array}$ & $\begin{array}{l}\text { Synthetic } \\
\text { data }\end{array}$ & $\begin{array}{l}\text { simulation- } \\
\text { based } \\
\text { implementat } \\
\text { ion in } \\
\text { Java }\end{array}$ & $\begin{array}{l}\text { HCLB } \\
\text { systematically } \\
\text { avoids } \\
\text { pushing } \\
\text { its compute } \\
\text { nodes into the } \\
\text { over utilized } \\
\text { state, } \\
\text { refraining so } \\
\text { from such } \\
\text { load } \\
\text { balancing- } \\
\text { induced } \\
\text { violations. }\end{array}$ \\
\hline $\begin{array}{l}\text { VM launching } \\
\text { overhead } \\
\text { reference } \\
\text { model (Wu et } \\
\text { al. [24]) }\end{array}$ & $\begin{array}{l}\text { The model can } \\
\text { accurately } \\
\text { predict } \\
\text { launching } \\
\text { overhead within } \\
\text { a mean square } \\
\text { weighted } \\
\text { deviation less } \\
\text { than three from } \\
\text { all four } \\
\text { variables, i.e. } \\
\text { VM CPU } \\
\text { utilization, } \\
\text { system CPU } \\
\text { utilization, } \\
\text { system I/O } \\
\text { utilization and } \\
\text { VM launching } \\
\text { time }\end{array}$ & $\begin{array}{l}\text { two types of } \\
\text { VM } \\
\text { instances, i.e., } \\
\text { a small } \\
\text { instance } \\
\text { configured } \\
\text { with one } \\
\text { virtual } \\
\text { CPU core and } \\
2 \text { GB memory, } \\
\text { and a large } \\
\text { instance } \\
\text { configured } \\
\text { with } 16 \text { virtual } \\
\text { CPU cores and } \\
32 \\
\text { memory GB }\end{array}$ & $\begin{array}{l}\text { Cloud } \\
\text { bursting }\end{array}$ & $\begin{array}{l}\text { Workload } \\
\text { traces } \\
\text { from } \\
\text { FermiClo } \\
\text { ud, a } \\
\text { private } \\
\text { cloud } \\
\text { developed } \\
\text { by the } \\
\text { Fermi } \\
\text { National } \\
\text { Accelerat } \\
\text { or } \\
\text { Laborator } \\
\text { y for } \\
\text { scientific } \\
\text { workflows } \\
\text {. }\end{array}$ & $\begin{array}{l}\text { Simulation } \\
\text { with } \\
\text { workload } \\
\text { traces from } \\
\text { FermiCloud }\end{array}$ & $\begin{array}{l}\text { With help } \\
\text { reference } \\
\text { model } \\
\text { efficient } \\
\text { resource } \\
\text { allocation } \\
\text { algorithms } \\
\text { can be } \\
\text { developed for } \\
\text { cloud bursting } \\
\text { process to } \\
\text { minimize the } \\
\text { operational } \\
\text { cost } \\
\text { resource and } \\
\text { waste. }\end{array}$ \\
\hline
\end{tabular}




\begin{tabular}{|c|c|c|c|c|c|c|}
\hline Scheme( Ref.) & Description & $\begin{array}{l}\text { Experimental } \\
\text { Configuration }\end{array}$ & $\begin{array}{l}\text { Technique } \\
\text { used }\end{array}$ & workload & Evaluation & $\begin{array}{l}\text { Performance } \\
\text { improvement }\end{array}$ \\
\hline $\begin{array}{l}\text { Brownout (Xu } \\
\text { et al. [25]) }\end{array}$ & $\begin{array}{l}\text { brownout } \\
\text { approach reduces } \\
\text { energy } \\
\text { consumption } \\
\text { through } \\
\text { selectively and } \\
\text { dynamically } \\
\text { deactivating } \\
\text { application } \\
\text { optional } \\
\text { components. }\end{array}$ & $\begin{array}{l}\text { Simulation } \\
\text { with } 100 \text { hosts. }\end{array}$ & $\begin{array}{l}\text { VM } \\
\text { placement } \\
\text { and } \\
\text { consolidation } \\
\text { algorithm }\end{array}$ & $\begin{array}{l}\text { Workload } \\
\text { traces } \\
\text { from } \\
\text { PlanetLab }\end{array}$ & $\begin{array}{l}\text { CloudSim } \\
\text { framework }\end{array}$ & $\begin{array}{l}\text { proposed } \\
\text { policies save } \\
\text { more energy } \\
\text { than the } \\
\text { baselines } \\
\text { PCO(placeme } \\
\text { nt and } \\
\text { consolidation } \\
\text { algorithm) } \\
\text { and } \\
\text { UBP(Utilizati } \\
\text { on-based } \\
\text { Probabilistic } \\
\text { VM } \\
\text { consolidation } \\
\text { algorithm) }\end{array}$ \\
\hline $\begin{array}{l}\text { M-convex VM } \\
\text { consolidation } \\
\text { (Huang et al. } \\
\text { [26]) }\end{array}$ & $\begin{array}{l}\text { VM } \\
\text { consolidation } \\
\text { framework based } \\
\text { on quasi M- } \\
\text { convex } \\
\text { optimization } \\
\text { framework can } \\
\text { achieve a } \\
\text { balance among } \\
\text { multiple } \\
\text { administrative } \\
\text { objectives (e.g., } \\
\text { power cost, } \\
\text { network cost) } \\
\text { during the VM } \\
\text { consolidation } \\
\text { process. }\end{array}$ & $\begin{array}{l}\text { Simulation } \\
\text { with } 550 \\
\text { servers }\end{array}$ & $\begin{array}{l}\text { VM } \\
\text { consolidation }\end{array}$ & $\begin{array}{l}\text { Workload } \\
\text { traces } \\
\text { from } \\
\text { PlanetLab }\end{array}$ & CloudSim & $\begin{array}{l}\text { the proposed } \\
\text { framework is } \\
\text { efficient, } \\
\text { scalable and } \\
\text { highly } \\
\text { practical. }\end{array}$ \\
\hline \begin{tabular}{l}
\multicolumn{3}{l}{ BGM-BLA } \\
$($ Tao et al. \\
$[27])$
\end{tabular} & $\begin{array}{l}\text { A binary graph } \\
\text { matching-based } \\
\text { bucket-code } \\
\text { learning } \\
\text { algorithm } \\
\text { (BGM-BLA) is } \\
\text { designed for } \\
\text { solving } \\
\text { dynamic the } \\
\text { migration of } \\
\text { VMs (DM-VM ) } \\
\text { problem. }\end{array}$ & 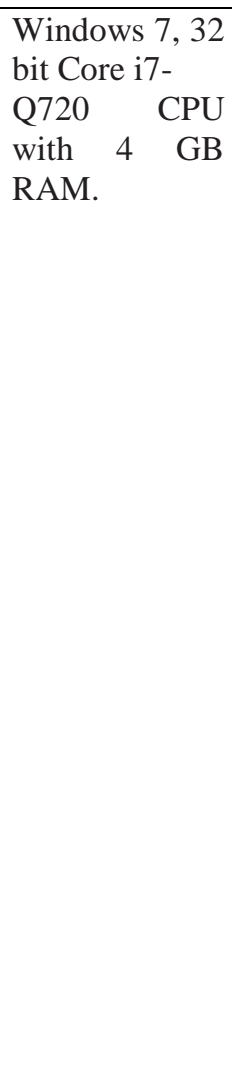 & $\begin{array}{l}\text { binary graph } \\
\text { matching- } \\
\text { based bucket- } \\
\text { code learning } \\
\text { algorithm }\end{array}$ & $\begin{array}{l}\text { Synthetic } \\
\text { data }\end{array}$ & Simulation & $\begin{array}{l}\text { BGM-BLA } \\
\text { algorithm } \\
\text { performs } \\
\text { relatively well } \\
\text { in terms of the } \\
\text { Pareto sets } \\
\text { obtained and } \\
\text { computational } \\
\text { time in } \\
\text { comparison } \\
\text { with two } \\
\text { optimization } \\
\text { algorithms, } \\
\text { i.e., } \\
\text { Non- } \\
\text { dominated } \\
\text { Sorting } \\
\text { Genetic } \\
\text { Algorithm } \\
\text { (NSGA-II) } \\
\text { and binary } \\
\text { graph } \\
\text { matching- } \\
\text { based } \\
\text { common- } \\
\text { coding } \\
\text { algorithm. }\end{array}$ \\
\hline
\end{tabular}




\begin{tabular}{|c|c|c|c|c|c|c|}
\hline Scheme( Ref.) & Description & $\begin{array}{l}\text { Experimental } \\
\text { Configuration }\end{array}$ & $\begin{array}{l}\text { Technique } \\
\text { used }\end{array}$ & workload & Evaluation & $\begin{array}{l}\text { Performance } \\
\text { improvement }\end{array}$ \\
\hline $\begin{array}{l}\text { MinES \& } \\
\text { MinCS (Dai et } \\
\text { al. [28]) }\end{array}$ & $\begin{array}{l}\text { two greedy } \\
\text { approximation } \\
\text { algorithms, } \\
\text { minimum energy } \\
\text { virtual machine } \\
\text { (VM) scheduling } \\
\text { algorithm } \\
\text { (MinES) and } \\
\text { minimum } \\
\text { communication } \\
\text { virtual machine } \\
\text { scheduling } \\
\text { algorithm } \\
\text { (MinCS), to } \\
\text { reduce the } \\
\text { energy while } \\
\text { satisfying the } \\
\text { tenants' service } \\
\text { level agreements. }\end{array}$ & $\begin{array}{l}\text { Dell } \\
\text { PowerEdge } \\
\text { Rack Servers }\end{array}$ & $\begin{array}{l}\text { greedy } \\
\text { approximatio } \\
\text { n. }\end{array}$ & $\begin{array}{l}\text { Synthetic } \\
\text { workloads } \\
\text { and } \\
\text { Google's } \\
\text { job traces }\end{array}$ & $\begin{array}{l}\text { Simulation } \\
\text { \& real test- } \\
\text { bed. }\end{array}$ & $\begin{array}{l}\text { results } \\
\text { demonstrated } \\
\text { that MinES } \\
\text { and MinCS } \\
\text { yield } \\
\text { scheduling } \\
\text { that are within } \\
4.3 \text { to } 6.1 \\
\text { percent } \\
\text { energy } \\
\text { consumption } \\
\text { of the optimal } \\
\text { solution while } \\
\text { being } \\
\text { computational } \\
\text { ly efficient }\end{array}$ \\
\hline $\begin{array}{l}\text { Platform for } \\
\text { virtual } \\
\text { machine (VM) } \\
\text { placement/mig } \\
\text { ration(VAKILI } \\
\text { NIA et al. } \\
[29])\end{array}$ & $\begin{array}{l}\text { Platform for } \\
\text { virtual machine } \\
\text { (VM) } \\
\text { placement/migrat } \\
\text { ion is proposed } \\
\text { to minimize the } \\
\text { total power } \\
\text { consumption of } \\
\text { cloud data } \\
\text { centers (DCs). }\end{array}$ & $\begin{array}{l}\text { Intel Xeon } \\
\text { Processor E5- } \\
2660 \text { v2 CPUs } \\
\text { and } \\
\text { 8x16GB } \\
\text { DDR3 } \\
\text { (M393B2G70 } \\
\text { DB0-CMA) } \\
\text { RAM }\end{array}$ & $\begin{array}{l}\text { (i)estimation } \\
\text { module based } \\
\text { on ARIMA } \\
\text { (Auto } \\
\text { Regressive } \\
\text { Integrated } \\
\text { Moving } \\
\text { Average) } \\
\text { model was } \\
\text { introduced to } \\
\text { predict the } \\
\text { incoming } \\
\text { load of the } \\
\text { DC } \\
\text { (ii) } \\
\text { schedulers } \\
\text { were } \\
\text { designed to } \\
\text { determine the } \\
\text { optimal } \\
\text { assignment of } \\
\text { VMs to the } \\
\text { PMs } \\
\text { (iii) Column } \\
\text { generation } \\
\text { (CG) method } \\
\text { was applied } \\
\text { to solve the } \\
\text { large-scale } \\
\text { optimization } \\
\text { problem. }\end{array}$ & $\begin{array}{l}\text { Synthetic } \\
\text { data }\end{array}$ & $\begin{array}{l}\text { MATLAB } \\
\text { and IBM } \\
\text { ILOG } \\
\text { CPLEX }\end{array}$ & $\begin{array}{l}\text { results have } \\
\text { shown that } \\
\text { the approach } \\
\text { explores the } \\
\text { optimal } \\
\text { solution with } \\
\text { an optimality } \\
\text { gap of at most } \\
1 \% \text { in } 3 \\
\text { minutes } \\
\text { Computation } \\
\text { time. }\end{array}$ \\
\hline
\end{tabular}




\begin{tabular}{|c|c|c|c|c|c|c|}
\hline Scheme( Ref.) & Description & $\begin{array}{l}\text { Experimental } \\
\text { Configuration }\end{array}$ & $\begin{array}{l}\text { Technique } \\
\text { used }\end{array}$ & workload & Evaluation & $\begin{array}{l}\text { Performance } \\
\text { improvement }\end{array}$ \\
\hline $\begin{array}{l}\text { Green-Works } \\
\text { (Li et al. [30]) }\end{array}$ & $\begin{array}{l}\text { Green-Works, a } \\
\text { framework for } \\
\text { HPC data centers } \\
\text { running on a } \\
\text { renewable } \\
\text { energy mix }\end{array}$ & $\begin{array}{l}\text { IBM } \\
\text { System x3650 } \\
\text { M2 \& Intel } \\
\text { Xeon X5570 }\end{array}$ & $\begin{array}{l}\text { Green- } \\
\text { Works, a } \\
\text { framework }\end{array}$ & $\begin{array}{l}\text { Real- } \\
\text { World } \\
\text { Workload } \\
\text { Traces }\end{array}$ & $\begin{array}{l}\text { Simulation } \\
\text { with MatLab }\end{array}$ & $\begin{array}{l}\text { Reduce } \\
\text { average } \\
\text { performance } \\
\text { degradation } \\
\text { by only } 60 \% \\
\text { and worst- } \\
\text { case } \\
\text { performance } \\
\text { degradation } \\
\text { by } 40 \% \text { while } \\
\text { maintaining a } \\
\text { desired } \\
\text { energy } \\
\text { efficiency, } \\
\text { battery } \\
\text { lifecycle, and } \\
\text { backup time. }\end{array}$ \\
\hline $\begin{array}{l}\text { Enhanced bee } \\
\text { colony } \\
\text { algorithm } \\
\text { (Babu et al. } \\
{[31] \text { ) }}\end{array}$ & $\begin{array}{l}\text { Enhanced bee } \\
\text { colony algorithm } \\
\text { for efficient and } \\
\text { effective load } \\
\text { balancing in } \\
\text { cloud } \\
\text { environment }\end{array}$ & $\begin{array}{l}\text { Simulation } \\
\text { with dynamic } \\
\text { environment }\end{array}$ & $\begin{array}{l}\text { bee colony } \\
\text { algorithm }\end{array}$ & $\begin{array}{l}\text { Synthetic } \\
\text { data }\end{array}$ & Simulation & $\begin{array}{l}\text { reduce the } \\
\text { imbalance in } \\
\text { the cloud eco } \\
\text { system }\end{array}$ \\
\hline $\begin{array}{l}\text { Dynamic VMs } \\
\text { placement } \\
\text { using PSO } \\
\text { (Dashti et al. } \\
{[32] \text { ) }}\end{array}$ & $\begin{array}{l}\text { PSO [Particle } \\
\text { Swarm } \\
\text { Optimization] to } \\
\text { guarantee } \\
\text { Quality of } \\
\text { service of users' } \\
\text { tasks, and } \\
\text { improve energy } \\
\text { efficiency of } \\
\text { cloud computing }\end{array}$ & NA & PSO & $\begin{array}{l}\text { Synthetic } \\
\text { data }\end{array}$ & CloudSim & $\begin{array}{l}\text { save as much } \\
\text { as } 14 \% \text { more } \\
\text { energy and } \\
\text { the number of } \\
\text { migrations }\end{array}$ \\
\hline $\begin{array}{l}\text { Fuzzy logic } \\
\text { and heuristic } \\
\text { based virtual } \\
\text { machine } \\
\text { consolidation( } \\
\text { Monil et } \\
\text { al.[33]) }\end{array}$ & $\begin{array}{l}\text { Fuzzy logic and } \\
\text { heuristic based } \\
\text { virtual machine } \\
\text { consolidation to } \\
\text { ensure energy- } \\
\text { QoS. balance. }\end{array}$ & $\begin{array}{l}\text { Simulation } \\
\text { with } 800 \\
\text { heterogeneous } \\
\text { physical } \\
\text { nodes. }\end{array}$ & $\begin{array}{l}\text { Fuzzy logic } \\
\text { and heuristic }\end{array}$ & $\begin{array}{l}\text { work load } \\
\text { data } \\
\text { provided } \\
\text { from } \\
\text { CoMon } \\
\text { project, a } \\
\text { monitorin } \\
\text { g } \\
\text { infrastruct } \\
\text { ure for } \\
\text { PlanetLab. }\end{array}$ & CloudSim & $\begin{array}{l}\text { Minimum } \\
\text { energy } \\
\text { consumption } \\
\text { by the } \\
\text { proposed } \\
\text { method is } 102 \\
\text { Kwh therefore } \\
\text { we got } 8.5 \% \\
\text { reduction as } \\
\text { compared to } \\
\text { baseline } \\
\text { algorithm. }\end{array}$ \\
\hline
\end{tabular}




\begin{tabular}{|c|c|c|c|c|c|c|}
\hline Scheme( Ref.) & Description & $\begin{array}{l}\text { Experimental } \\
\text { Configuration }\end{array}$ & $\begin{array}{l}\text { Technique } \\
\text { used }\end{array}$ & workload & Evaluation & $\begin{array}{l}\text { Performance } \\
\text { improvement }\end{array}$ \\
\hline $\begin{array}{lr}\text { Jettison } & \text { for } \\
\text { partial } & \text { VM } \\
\text { migration } & \\
\text { (BILA et al. } \\
{[34] \text { ) }} & \end{array}$ & $\begin{array}{l}\text { An approach that } \\
\text { transparently } \\
\text { migrates only the } \\
\text { working set of an } \\
\text { idle VM onto the } \\
\text { consolidation } \\
\text { server by } \\
\text { copying only } \\
\text { VM metadata. } \\
\text { places desktop } \\
\text { PCs in low- } \\
\text { power mode } \\
\text { when inactive } \\
\text { and switches } \\
\text { them to running } \\
\text { mode when } \\
\text { pages are needed } \\
\text { by the VM } \\
\text { running on the } \\
\text { consolidation } \\
\text { server }\end{array}$ & $\begin{array}{l}\text { Power Profile } \\
\text { of Dell Studio } \\
\text { XPS } 7100 \text { PC }\end{array}$ & $\begin{array}{l}\text { sleep } \\
\text { scheduling } \\
\text { and } \\
\text { prefetching } \\
\text { algorithms }\end{array}$ & $\begin{array}{l}\text { real user } \\
\text { traces } \\
\text { collected } \\
\text { using a } \\
\text { Mac OS } \\
\text { X-based } \\
\text { tracker. }\end{array}$ & $\begin{array}{ll}\text { Real } \& \\
\text { prototype } \\
\text { based }\end{array}$ & $\begin{array}{l}\text { Jettison can } \\
\text { deliver } 44- \\
91 \% \text { energy } \\
\text { savings } \\
\text { during idle } \\
\text { periods of at } \\
\text { least } 10 \\
\text { minutes, } \\
\text { while } \\
\text { providing low } \\
\text { migration } \\
\text { latencies of } \\
\text { about } \\
\text { seconds and } \\
\text { migrating } \\
\text { minimal state } \\
\text { that is under } \\
\text { an order of } \\
\text { magnitude of } \\
\text { the VM's } \\
\text { memory } \\
\text { footprint. }\end{array}$ \\
\hline $\begin{array}{l}\text { Energy-aware } \\
\text { resource } \\
\text { provisioning } \\
\text { framework } \\
\text { (Dabbagh et al. } \\
\text { [35]) }\end{array}$ & 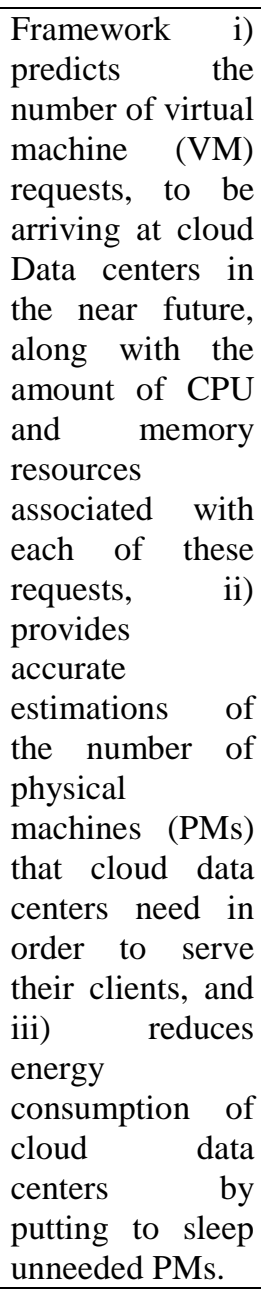 & $\begin{array}{l}\text { Intel Core } 2 \\
\text { Quad Q9400 } \\
2.66 \quad \text { GHz } \\
\text { processor, and } \\
\text { an 8 GB RAM. }\end{array}$ & $\begin{array}{l}\text { modified Best } \\
\text { Fit } \\
\text { Decreasing } \\
\text { (BFD) } \\
\text { heuristic for } \\
\text { power } \\
\text { management }\end{array}$ & $\begin{array}{l}\text { real } \\
\text { Google } \\
\text { traces }\end{array}$ & $\begin{array}{l}\text { Real \& } \\
\text { simulation }\end{array}$ & $\begin{array}{l}\text { Proposed } \\
\text { energy-aware } \\
\text { resource } \\
\text { provisioning } \\
\text { framework } \\
\text { makes } \\
\text { substantial } \\
\text { energy } \\
\text { savings. }\end{array}$ \\
\hline
\end{tabular}




\begin{tabular}{|c|c|c|c|c|c|c|}
\hline Scheme( Ref.) & Description & $\begin{array}{l}\text { Experimental } \\
\text { Configuration }\end{array}$ & $\begin{array}{l}\text { Technique } \\
\text { used }\end{array}$ & workload & Evaluation & $\begin{array}{l}\text { Performance } \\
\text { improvement }\end{array}$ \\
\hline $\begin{array}{l}\text { ACS-VMC } \\
\text { (Farahnakian } \\
\text { et al. [36]) }\end{array}$ & $\begin{array}{l}\text { Ant Colony } \\
\text { System (ACS) } \\
\text { based VM } \\
\text { Consolidation } \\
\text { (ACS-VMC) } \\
\text { approach to } \\
\text { reduces energy } \\
\text { consumption } \\
\text { while } \\
\text { maintaining the } \\
\text { required } \\
\text { performance } \\
\text { levels in a cloud } \\
\text { data center }\end{array}$ & $\begin{array}{l}\text { Simulation } \\
\text { with } 800 \\
\text { heterogeneous } \\
\text { PMs }\end{array}$ & $\begin{array}{l}\text { Ant Colony } \\
\text { System }\end{array}$ & $\begin{array}{l}\text { Real } \\
\text { workload } \\
\text { from } \\
\text { CoMon } \\
\text { project, a } \\
\text { monitorin } \\
\text { g } \\
\text { infrastruct } \\
\text { ure for } \\
\text { PlanetLab. }\end{array}$ & Simulation & $\begin{array}{l}\text { ACS-VMC } \\
\text { consumes less } \\
\text { ESV(Energy } \\
\text { and SLA } \\
\text { Violations) } \\
\text { than other } \\
\text { benchmarks } \\
\text { algorithms in } \\
\text { the real } \\
\text { workload } \\
\text { traces. }\end{array}$ \\
\hline $\begin{array}{l}\text { SmartSLA } \\
\text { (Xiong et al. } \\
[37])\end{array}$ & $\begin{array}{l}\text { a cost-sensitive } \\
\text { virtualized } \\
\text { resource } \\
\text { management } \\
\text { system for CPU- } \\
\text { bound database } \\
\text { services. } \\
\text { SmartSLA } \\
\text { minimizes the } \\
\text { total cost for a } \\
\text { DBaaS provider } \\
\text { which is } \\
\text { composed of } \\
\text { SLA penalty } \\
\text { cost. }\end{array}$ & $\begin{array}{l}\text { AMD } 3.0 \mathrm{GHz} \\
\text { Dual cores \& } \\
\text { Intel Xeon } \\
2.40 \quad \text { GHz } \\
\text { Hexa-core }\end{array}$ & $\begin{array}{l}\text { machine } \\
\text { learning } \\
\text { technologies }\end{array}$ & $\begin{array}{l}\text { web traces } \\
\text { from the } \\
1998 \\
\text { World } \\
\text { Cup site. }\end{array}$ & $\begin{array}{l}\text { Real } \\
\text { environment }\end{array}$ & $\begin{array}{l}\text { SmartSLA is } \\
\text { able to } \\
\text { minimize the } \\
\text { total cost } \\
\text { under time- } \\
\text { varying } \\
\text { workloads } \\
\text { compared to } \\
\text { the other cost- } \\
\text { insensitive } \\
\text { approaches. }\end{array}$ \\
\hline $\begin{array}{l}\text { VM workload } \\
\text { cycles } \\
\text { (Baruchi et al. } \\
\text { [38]) }\end{array}$ & $\begin{array}{l}\text { VM workload an } \\
\text { important factor } \\
\text { and carefully } \\
\text { choosing } \\
\text { proper moment } \\
\text { to migrate a VM } \\
\text { can reduce the } \\
\text { live migration } \\
\text { penalties. }\end{array}$ & Simulation & $\begin{array}{l}\text { VM } \\
\text { migration }\end{array}$ & $\begin{array}{l}\text { Synthetic } \\
\text { data }\end{array}$ & $\begin{array}{l}\text { Simulation } \\
\text { on cloudsim }\end{array}$ & $\begin{array}{l}\text { Reduce up to } \\
43 \% \text { of } \\
\text { network data } \\
\text { transfer and } \\
\text { reduce up to } \\
74 \% \text { of live } \\
\text { migration } \\
\text { time when } \\
\text { compared to } \\
\text { traditional } \\
\text { consolidation } \\
\text { strategies }\end{array}$ \\
\hline $\begin{array}{l}\text { HGA for the } \\
\text { VM placement } \\
\text { (Tang et al. } \\
\text { [39]) }\end{array}$ & $\begin{array}{l}\text { HGA( Hybrid } \\
\text { genetic } \\
\text { algorithm )for a } \\
\text { new virtual } \\
\text { machine } \\
\text { placement } \\
\text { problem that } \\
\text { considered the } \\
\text { energy } \\
\text { consumption in } \\
\text { both physical } \\
\text { machines and the } \\
\text { communication } \\
\text { network in a data } \\
\text { center }\end{array}$ & $\begin{array}{l}\text { Intel Core } 2 \\
\text { Duo CPU of } \\
\text { 3.00GHz and a } \\
\text { 4.00GB RAM. }\end{array}$ & $\begin{array}{l}\text { Hybrid } \\
\text { Genetic } \\
\text { Algorithm }\end{array}$ & $\begin{array}{l}\text { Synthetic } \\
\text { data }\end{array}$ & $\begin{array}{l}\text { Simulation } \\
\text { using java }\end{array}$ & $\begin{array}{l}\text { Hybrid } \\
\text { genetic } \\
\text { algorithm } \\
\text { significantly } \\
\text { outperforms } \\
\text { the original } \\
\text { genetic } \\
\text { algorithm, } \\
\text { and that the } \\
\text { hybrid genetic } \\
\text { algorithm is } \\
\text { scalable. }\end{array}$ \\
\hline
\end{tabular}




\begin{tabular}{|c|c|c|c|c|c|c|}
\hline Scheme( Ref.) & Description & $\begin{array}{l}\text { Experimental } \\
\text { Configuration }\end{array}$ & $\begin{array}{l}\text { Technique } \\
\text { used }\end{array}$ & workload & Evaluation & $\begin{array}{l}\text { Performance } \\
\text { improvement }\end{array}$ \\
\hline $\begin{array}{l}\text { Energy aware } \\
\text { profiling(Alza } \\
\text { mil et al. [40]) }\end{array}$ & $\begin{array}{l}\text { Energy aware } \\
\text { profiling helps us } \\
\text { to better } \\
\text { understand the } \\
\text { way the energy } \\
\text { is consumed and } \\
\text { help the } \\
\text { application } \\
\text { developer from } \\
\text { the application } \\
\text { level enhance } \\
\text { their decision- } \\
\text { making in terms } \\
\text { of energy- } \\
\text { awareness when } \\
\text { optimizing their } \\
\text { applications and } \\
\text { services. }\end{array}$ & $\begin{array}{l}\text { Four Dell } \\
\text { commodity } \\
\text { servers(server } \\
\text { consists of a } \\
\text { four core } \\
\text { X3430 Intel } \\
\text { Xeon CPU, } \\
\text { running } \\
\text { at the default } \\
\text { clock speed of } \\
\text { 2.40GHz and a } \\
\text { total of 8GB of } \\
\text { RAM ) }\end{array}$ & $\begin{array}{l}\text { Energy aware } \\
\text { profiling }\end{array}$ & $\begin{array}{l}\text { Leeds } \\
\text { Testbed }\end{array}$ & $\begin{array}{l}\text { Real \& } \\
\text { simulation }\end{array}$ & $\begin{array}{l}\text { energy- } \\
\text { awareness at } \\
\text { physical host } \\
\text { and virtual } \\
\text { machine } \\
\text { levels. }\end{array}$ \\
\hline $\begin{array}{l}\text { PRS(Chen et } \\
\text { al. [41]) }\end{array}$ & 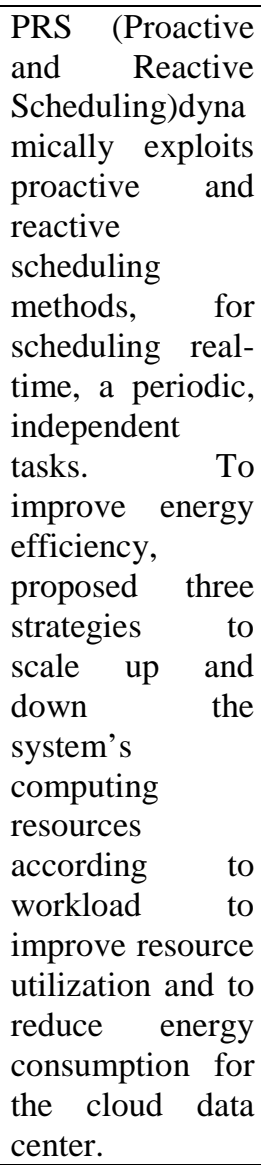 & $\begin{array}{l}\text { KVM cluster } \\
\text { with five Dell } \\
\text { Optiplex } \\
7010 \mathrm{MT} \text {, each } \\
\text { of physical } \\
\text { host has a } \\
\text { CPU (i3 } \\
\begin{array}{l}\text { 3.9GHz } \\
\text { 4cores), 3.7G } \\
\text { memory }\end{array}\end{array}$ & $\begin{array}{l}\text { interval } \\
\text { number } \\
\text { theory }\end{array}$ & $\begin{array}{l}\text { Synthetic } \\
\text { workloads } \\
\text { and } \\
\text { Google } \\
\text { workload } \\
\text { traces. }\end{array}$ & $\begin{array}{l}\text { Real } \\
\text { environment } \\
\& \\
\text { simulation } \\
\text { with } \\
\text { cloudsim }\end{array}$ & $\begin{array}{l}\text { PRS performs } \\
\text { better than } \\
\text { baseline } \\
\text { algorithms, } \\
\text { and can } \\
\text { effectively } \\
\text { improve the } \\
\text { performance } \\
\text { of a cloud } \\
\text { data center. }\end{array}$ \\
\hline
\end{tabular}




\begin{tabular}{|c|c|c|c|c|c|c|}
\hline Scheme( Ref.) & Description & $\begin{array}{l}\text { Experimental } \\
\text { Configuration }\end{array}$ & $\begin{array}{l}\text { Technique } \\
\text { used }\end{array}$ & workload & Evaluation & $\begin{array}{l}\text { Performance } \\
\text { improvement }\end{array}$ \\
\hline $\begin{array}{l}\text { Dynamic } \\
\text { Resource } \\
\text { Allocation } \\
\text { using Virtual } \\
\text { Machines } \\
\text { (Xiao et al. } \\
\text { [42]) }\end{array}$ & 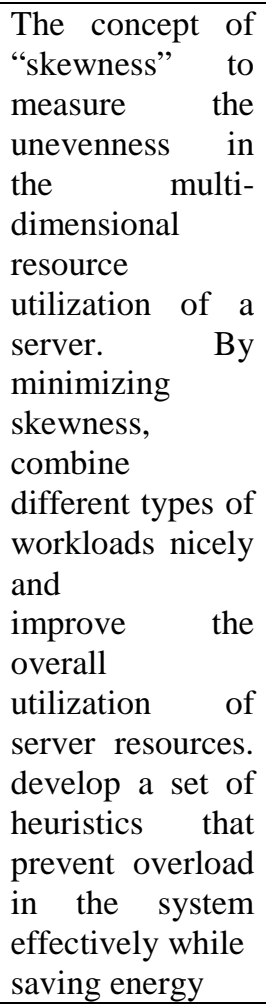 & $\begin{array}{l}30 \text { Dell } \\
\text { PowerEdge } \\
\text { blade servers } \\
\text { with Intel } \\
\text { E5620 CPU } \\
\text { and 24GB } \\
\text { of RAM. }\end{array}$ & skewness & $\begin{array}{l}\text { traces } \\
\text { from } \\
\text { servers } \\
\text { and } \\
\text { desktop } \\
\text { computers } \\
\text { in } \\
\text { university }\end{array}$ & simulation & $\begin{array}{l}\text { Achieves both } \\
\text { overload } \\
\text { avoidance and } \\
\text { green } \\
\text { computing for } \\
\text { systems with } \\
\text { multi- } \\
\text { resource } \\
\text { constraints. }\end{array}$ \\
\hline $\begin{array}{l}\text { MHOD-OPT } \\
\text { (Baloglazov et } \\
\text { al. [43]) }\end{array}$ & $\begin{array}{l}\text { Optimal Markov } \\
\text { Host } \\
\text { Overload } \\
\text { Detection } \\
\text { (MHOD-OPT) } \\
\text { algorithm } \\
\text { for the problem } \\
\text { of host } \\
\text { overload } \\
\text { detection as a } \\
\text { part of dynamic } \\
\text { VM } \\
\text { consolidation. }\end{array}$ & simulation & $\begin{array}{l}\text { Markov chain } \\
\text { model }\end{array}$ & $\begin{array}{l}\text { PlanetLab } \\
\text { Workload } \\
\text { Traces }\end{array}$ & $\begin{array}{l}\text { Simulation } \\
\text { with } \\
\text { cloudsim }\end{array}$ & $\begin{array}{l}\text { Proved best } \\
\text { among } \\
\text { baseline } \\
\text { techniques }\end{array}$ \\
\hline $\begin{array}{l}\text { Novel adaptive } \\
\text { heuristics for } \\
\text { dynamic } \\
\text { consolidation } \\
\text { of VMs } \\
\text { (Beloglazov et } \\
\text { al. [44]) }\end{array}$ & $\begin{array}{l}\text { novel adaptive } \\
\text { heuristics for } \\
\text { dynamic } \\
\text { onsolidation of } \\
\text { VMs based on an } \\
\text { analysis of } \\
\text { historical data } \\
\text { from reme } \\
\text { resource usage } \\
\text { by VMs. }\end{array}$ & $\begin{array}{l}\text { Simulation } \\
\text { with } 800 \\
\text { heterogeneous } \\
\text { physical nodes }\end{array}$ & $\begin{array}{l}\text { adaptive } \\
\text { heuristics }\end{array}$ & $\begin{array}{l}\text { Real } \\
\text { workload } \\
\text { from } \\
\text { CoMon } \\
\text { project, a } \\
\text { monitorin } \\
\text { g } \\
\text { infrastruct } \\
\text { ure for } \\
\text { PlanetLab }\end{array}$ & $\begin{array}{l}\text { CloudSim } \\
\text { toolkit }\end{array}$ & $\begin{array}{l}\text { significantly } \\
\text { reduced } \\
\text { energy } \\
\text { consumption, } \\
\text { while } \\
\text { ensuring a } \\
\text { high level of } \\
\text { adherence to } \\
\text { the Service } \\
\text { Level } \\
\text { Agreements }\end{array}$ \\
\hline
\end{tabular}




\begin{tabular}{|c|c|c|c|c|c|c|}
\hline Scheme( Ref.) & Description & $\begin{array}{l}\text { Experimental } \\
\text { Configuration }\end{array}$ & $\begin{array}{l}\text { Technique } \\
\text { used }\end{array}$ & workload & Evaluation & $\begin{array}{l}\text { Performance } \\
\text { improvement }\end{array}$ \\
\hline \begin{tabular}{l}
\multicolumn{3}{l}{\begin{tabular}{l}
\multicolumn{2}{l}{ VM placement } \\
(Wu et al. \\
$[45])$
\end{tabular}} \\
\end{tabular} & $\begin{array}{l}\text { genetic } \\
\text { algorithm for a } \\
\text { new virtual } \\
\text { machine } \\
\text { placement } \\
\text { problem that } \\
\text { considers the } \\
\text { energy } \\
\text { consumption in } \\
\text { both the servers } \\
\text { and the } \\
\text { communication } \\
\text { network in the } \\
\text { data center. }\end{array}$ & Simulation & $\begin{array}{l}\text { genetic } \\
\text { algorithm }\end{array}$ & $\begin{array}{l}\text { synthetic } \\
\text { workloads }\end{array}$ & $\begin{array}{l}\text { GA has been } \\
\text { implemente } \\
\text { d in Java. }\end{array}$ & $\begin{array}{l}\text { On average } \\
\text { the solutions } \\
\text { produced by } \\
\text { the GA are } \\
\text { 3:5\%-23:5\% } \\
\text { better than } \\
\text { those } \\
\text { produced by } \\
\text { the FFD(First } \\
\text { Fit } \\
\text { Decreasing). }\end{array}$ \\
\hline $\begin{array}{l}\text { DCeP (Sego et } \\
\text { al. [46]) }\end{array}$ & $\begin{array}{l}\text { Data Center } \\
\text { Energy } \\
\text { Productivity } \\
\text { [DCeP] metric, } \\
\text { which is the ratio } \\
\text { of useful work } \\
\text { produced by the } \\
\text { data center to the } \\
\text { energy, } \\
\text { consumed } \\
\text { performing that } \\
\text { work. }\end{array}$ & $\begin{array}{l}\text { Pacific } \\
\text { Northwest } \\
\text { National } \\
\text { Laboratory } \\
\text { (PNNL) }\end{array}$ & $\begin{array}{l}\text { Data Center } \\
\text { Energy } \\
\text { Productivity }\end{array}$ & $\begin{array}{l}\text { HPC } \\
\text { Workload }\end{array}$ & $\begin{array}{l}\text { Real } \\
\text { environment }\end{array}$ & $\begin{array}{l}\text { DCeP can be } \\
\text { used to } \\
\text { clearly } \\
\text { measure and } \\
\text { distinguish } \\
\text { the energy } \\
\text { productivity } \\
\text { of different } \\
\text { operational } \\
\text { states in a } \\
\text { data center. }\end{array}$ \\
\hline $\begin{array}{l}\text { Barely alive } \\
\text { states } \\
\text { (Anagnostopo } \\
\text { ulou et al. } \\
{[47] \text { ) }}\end{array}$ & $\begin{array}{l}\text { Barely alive } \\
\text { states through } \\
\text { which we can } \\
\text { perform energy } \\
\text { efficient cloud } \\
\text { computing a } \\
\text { family of barely } \\
\text { alive active low- } \\
\text { power server } \\
\text { states that } \\
\text { facilitates both } \\
\text { fast reactivation } \\
\text { and access to } \\
\text { memory while in } \\
\text { a low-power } \\
\text { state. }\end{array}$ & $\begin{array}{l}\text { Simulation } \\
\text { with 32-node } \\
\text { clusters }\end{array}$ & $\begin{array}{l}\text { Barely alive } \\
\text { states }\end{array}$ & $\begin{array}{l}\text { Traces } \\
\text { from } \\
\text { Ask.com }\end{array}$ & Simulation &  \\
\hline $\begin{array}{l}\text { CADE(Kaplan } \\
\text { et al. [48]) }\end{array}$ & $\begin{array}{l}\text { CADE } \\
\text { [Corporate } \\
\text { Average } \\
\text { Datacenter } \\
\text { Efficiency], a } \\
\text { new industry } \\
\text { standard } \\
\text { efficiency } \\
\text { measure } \\
\text { developed by } \\
\text { McKinsey \& } \\
\text { Company. } \\
\end{array}$ & NA & CADE & NA & NA & $\begin{array}{l}\text { CADE helps } \\
\text { in calculating } \\
\text { energy } \\
\text { efficiency of a } \\
\text { data center. }\end{array}$ \\
\hline $\begin{array}{l}\text { VPM (Nathuji } \\
\text { et al. [49]) }\end{array}$ & $\begin{array}{l}\text { VirtualPower } \\
\text { Management } \\
\text { (VPM) approach } \\
\text { to online power } \\
\text { management }\end{array}$ & $\begin{array}{l}\text { multiple dual } \\
\text { core Pentium } 4 \\
\text { ma- } \\
\text { chines }\end{array}$ & virtualization & $\begin{array}{l}\text { Traces } \\
\text { from } \\
\text { Delta Air } \\
\text { Lines }\end{array}$ & $\begin{array}{l}\text { Real test- } \\
\text { bed }\end{array}$ & $\begin{array}{l}\text { VPM provide } \\
\text { up to } 34 \% \\
\text { improvements } \\
\text { in power } \\
\text { consumption. }\end{array}$ \\
\hline
\end{tabular}




\begin{tabular}{|c|c|c|c|c|c|c|}
\hline Scheme( Ref.) & Description & $\begin{array}{l}\text { Experimental } \\
\text { Configuration }\end{array}$ & $\begin{array}{l}\text { Technique } \\
\text { used }\end{array}$ & workload & Evaluation & $\begin{array}{l}\text { Performance } \\
\text { improvement }\end{array}$ \\
\hline $\begin{array}{l}\text { Thermal load } \\
\text { balancing } \\
\text { (Sharma et al. } \\
\text { [50]) }\end{array}$ & $\begin{array}{l}\text { local and } \\
\text { regional policies } \\
\text { for thermal } \\
\text { control for } \\
\text { reducing energy } \\
\text { consumption in } \\
\text { data center }\end{array}$ & $\begin{array}{l}\text { data center } \\
\text { with with } \\
\text { standard racks, } \\
\text { each } \\
\text { containing 20, } \\
\text { 2U (90mm) } \\
\text { high, Hewlett- } \\
\text { Packard A- } \\
\text { Class servers }\end{array}$ & $\begin{array}{l}\text { thermal load } \\
\text { balancing }\end{array}$ & $\begin{array}{l}\text { Real } \\
\text { traces }\end{array}$ & $\begin{array}{l}\text { fault } \\
\text { injection } \\
\text { simulations }\end{array}$ & \begin{tabular}{l}
\multicolumn{2}{l}{$\begin{array}{l}\text { energy } \\
\text { consumption } \\
\text { can }\end{array}$} \\
reduced by \\
more than \\
$14 \%$ by \\
workload \\
placement
\end{tabular} \\
\hline $\begin{array}{l}\text { Energy } \\
\text { efficient server } \\
\text { cluster(Elnoza } \\
\text { hy et al. [51]) }\end{array}$ & $\begin{array}{l}\text { five Server } \\
\text { power } \\
\text { management } \\
\text { technique, these } \\
\text { are Independent } \\
\text { voltage scaling } \\
\text { [IVS], } \\
\text { Coordinated } \\
\text { voltage scaling } \\
\text { [CVS], Vary-On } \\
\text { Vary-Off } \\
\text { [VOVO], } \\
\text { Combined policy } \\
\text { \& Coordinated } \\
\text { Policy to } \\
\text { perform energy } \\
\text { efficient cloud } \\
\text { computing }\end{array}$ & Simulation & $\begin{array}{l}\text { Server power } \\
\text { management } \\
\text { techniques }\end{array}$ & $\begin{array}{l}\text { Nagano } \\
\text { Winter } \\
\text { Olympics } \\
\text { Servers at } \\
\text { Columbus } \\
\text {. }\end{array}$ & Simulation & $\begin{array}{l}\text { IVS can only } \\
\text { account for } \\
\text { energy saving } \\
\text { of 20-30\%. } \\
\text { VOVO-CVS } \\
\text { combine help } \\
\text { to save up to } \\
18 \% \text { more } \\
\text { energy than } \\
\text { VOVO alone } \\
\text { but certainly } \\
\text { not without } \\
\text { complicated } \\
\text { implementatio } \\
\text { n. }\end{array}$ \\
\hline
\end{tabular}

\section{CONCLUSION AND FUTURE DIRECTIONS}

Multi tenancy, concurrency and distribution are main feature of any cloud computing architecture [52]. This paper covers different Vm Migration Algorithms for energy reduction in cloud computing (Heuristic ,Meta-heuristic\& Hybrid algorithms) along with other novel techniques. The main plus point of heuristic algorithms is that they give satisfactory solution to a problem in limited time cost frame. Heuristic algorithms are easier to implement in comparison to meta-heuristic algorithms. In Hybrid algorithms, heuristic algorithms are used to provide initial VM placement and meta-heuristic algorithms provides optimum placement of VMs during migration. This algorithm increases the implementation complexity but reduces time and cost space. Depending upon the kind of problem, you can use one or other. Now, we discuss the future directions and challenges as below:

- We observed that most meta-heuristic s gives better results in terms in energy consumption and QoS(Quality-of-service) but they are tested using only simulators. There is need to test these Meta-heuristic algorithms like genetic algorithms, ACO ( Ant colony optimization ,Particle Swarm Optimization and honey bee foraging algorithms in realistic environment. So that cloud vendors can adopt these methods to reduce energy consumption \& ensure QoS of user’s tasks.

- We also have observed that how it is difficult to ensure a balance between energy consumption and SLA violations. Therefore reducing energy consumption while maintain a QoS (Quality-of-Service) is a future research challenge.

- While considering the diversity of our surveyed papers, we want to know which algorithm is best under which environment. This question still is open because of heterogeneity of different algorithms and lack of validation of these algorithms under different realistic environment. A thorough testing of all these algorithms under same environment and under heterogeneous environment is definitely required as future work.

\section{ACKNOWLEDGMENT}

I would like to show my gratitude to Prof. Deepak Garg, Professor and Head Department of Computer Science and Engineering, Bennett University, Greater Noida, U.P. \& Nishi Goyal, Programmer at Panjab University, Chandigarh for providing me help \& support.

\section{REFERENCES}

[1] Dhir V. et. al., Internet of things: Vision, Challenges and Future Scope, International Journal of Advanced Research in Computer Science, May 2017.

[2] Singh N., Dhir V., Building Private Cloud Using Eucalyptus, Proceeding of $1^{\text {st }}$ National Conference on Emerging Trends on Engineering \& Technology (ETET- 2017) at SBBS University, Punjab. International Journal of Advanced 
Research in Computer Science, Vol. 8, No. 4, May 2017.

[3] Sigh N., Bal J.S., Energy Efficient Data Center: A systematic literature review, Advances in Computational Science and Technology, Research India Publications, Vol.10, Number 1, pp. 121-128, 2017, ISSN 0973-6107.

[4]. J. M. Kaplan, W. Forrest, and N. Kindler, "Revolutionizing data center energy efficiency,”Technical Report, available at http://www.mckinsey.com-/clientservice/bto/pointofview/pdf/-revolutionizing data center efficiency.pdf. Accessed on 25-06-2017.

[5] Dhir V. et. al., A Review on Image Forgery \& its Detection Procedure, International Journal of Advanced Research in Computer Science, May 2017.

[6]. Baloglazov A., (Thesis) Energy-Efficient anagement of Virtual Machines in Data Centers for Cloud Computing, THE UNIVERSITY OF MELBOURNE, 2013

[7]. Coffman Jr EG, Garey MR, Johnson DS. Approximation algorithms for bin packing: A survey. Approximation Algorithms for NP-Hard Problems. Boston, MA, USA: PWS Publishing Co.; 1996:46-93.

[8]. Talbi EG. Metaheuristics: From Design to Implementation, vol. 74: Hoboken, New Jersey, United States: John Wiley \& Sons; 2009.

[9]. Song X, Ma Y, Teng D. A load balancing scheme using federate migration based on virtual machines for cloud simulations. Math Prob Eng. 2015;2015:1-

[10]. Ni J, Huang Y, Luan Z, Zhang J, Qian D. Virtual machine mapping policy based on load balancing in private cloud environment. 2011 International Conference on Cloud and Service Computing (CSC). IEEE; 2011:292-295.

[11]. Tian W, Zhao Y, Zhong Y, Xu M, Jing C. A dynamic and integrated load-balancing scheduling algorithm for cloud datacenters. 2011 IEEE International Conference on Cloud Computing and Intelligence Systems. Beijing, China: IEEE; 2011:311-315.

[12]. Thiruvenkadam T, Kamalakkannan P. Energy efficient multi dimensional host load aware algorithm for virtual machine placement and optimization in cloud environment. Indian $\mathrm{J}$ Sci.

[13] WEN Y., LI Z., JIN S., LIN C., LIU Z., Energy-Efficient Virtual Resource Dynamic Integration Method in Cloud Computing, IEEE Special Section On Emerging Trends, Issues, And Challenges In Energy-Efficient Cloud Computing, June 29, 2017.

[14] WANG S, QIAN Z , YUAN J, YOU I, A DVFS Based Energy-Efficient Tasks Scheduling in a Data Center, IEEE Special Section On Emerging Trends, Issues, And Challenges In Energy-Efficient Cloud Computing, July 11, 2017,

[15] Laredo J.L.J., Guinand F.,Olivier D., Bouvry P., Load Balancing at the Edge of Chaos: How Self-Organized Criticality Can Lead to Energy-Efficient Computing, IEEE Transactions On Parallel And Distributed Systems, Vol. 28, No. 2, February 2017.

[16] Son J, Dastjerdi AV, Calheiros RN, Buyya R, SLA-Aware and Energy-Efficient Dynamic Overbooking in SDN-Based Cloud Data Centers, IEEE Transactions On Sustainable Computing, Vol. 2, No. 2, April-June 2017.

[17] ZHENG. H., FENG Y., TAN J., A Hybrid Energy-Aware Resource Allocation Approach in Cloud Manufacturing Environment, IEEE Special Section On Emerging CloudBased Wireless Communications And Networks, June 2017.

[18] Wang et al, Multiagent-Based Resource Allocation for Energy Minimization in Cloud Computing Systems, IEEE Transactions On Systems, Man, And Cybernetics: Systems, VOL. 47, NO. 2, February 2017.

[19] Minarolli D., Mazrekaj A., Freisleben B., Tracking uncertainty in long-term predictions for host overload and underload detection in cloud computing. Journal of
Cloud Computing Advances, Systems and Applications, Springer, 2017.

[20]. Ashraf A., Porres I., Multi-objective dynamic virtual machine consolidation in the cloud using ant colony system, International Journal of Parallel, Emergent and Distributed Systems, Taylor \&Francis, 2017.

[21] POOLA D., RAMAMOHANARAO K., BUYYA R., Enhancing Reliability of Workflow Execution Using Task Replication and Spot Instances, ACM Transactions on Autonomous and Adaptive Systems, Vol. 10, No. 4, Article 30,February 2016.

[22] Hieu N. T., Francesco M. D, Yl a-Jaaski A., Virtual Machine Consolidation with Multiple Usage Prediction for EnergyEfficient Cloud Data Centers, IEEE Transaction On Services Computing, March 2016.

[23] Pantazoglou M., Tzortzakis G., Delis A., Decentralized and Energy-Efficient Workload Management in Enterprise Clouds, IEEE Transactions On Cloud Computing, Vol. 4, No. 2, April-June 2016

[24] Wu H, Ren S, Garzoglio G, Timm S., Bernabeu G., Chadwick K.,Noh S-Y, A Reference Model for Virtual Machine Launching Overhead, IEEE Transactions On Cloud Computing, Vol. 4, No. 3, July-September 2016.

[25] Xu M., Dastjerdi A.V., Buyya R., Energy Efficient Scheduling of Cloud Application Components with Brownout, IEEE Transactions On Sustainable Computing, Vol. 1, No. 2, July-December 2016.

[26] Huang Z. et al, M-Convex VM Consolidation: Towards a Better VM Workload consolidation, IEEE Transactions On Cloud Computing, Vol. 4, No. 4, October- December 2016.

[27] Tao F. et al, BGM-BLA: A New Algorithm for Dynamic Migration of Virtual Machines in Cloud Computing, IEEE Transactions On Services Computing, Vol. 9, No. 6, November/December 2016.

[28] Dai X. et al, Energy-Efficient Virtual Machines Scheduling in Multi-Tenant Data Centers, IEEE Transactions On Cloud Computing, VOL. 4, NO. 2, APRIL-JUNE 2016.

[29] VAKILINIA S. et al , Energy Efficient Resource Allocation in Cloud Computing Environments, IEEE Special Section On Future Networks: Architectures, Protocols, And Applications, December 1, 2016.

[30] LI C., Wang R., Qian D., Li T., Managing Server Clusters on Renewable Energy Mix. ACM Transactions on Autonomous and Adaptive Systems, ACM, 2016.

[31]. Babu K.R.R., Samuel P, Enhanced Bee Colony Algorithm for Efficient Load Balancing and Scheduling in Cloud, Innovations in Bio-Inspired Computing and Applications, vol 424, pp 67-78, springer, 2016

[32] Dashti S.E., Rahmani A.M., Dynamic VMs placement for energy efficiency by PSO in cloud computing. Journal of Experimental \& Theoretical Artificial Intelligence, [Taylor \&Francis, 2016].

[33] Monil A. H., Rahman R. M., VM consolidation approach based on heuristics, fuzzy logic, and migration control. Journal of Cloud Computing Advances, Systems and Applications, Springer, 2016.

[34] BILA N., WRIGHT E.J., LARA E.D., JOSHI K., LAGARCAVILLA H.A., PARK E., GOEL A., TUNEN M.H., SATYANARAYANAN M., Energy-Oriented Partial Desktop Virtual Machine Migration, ACM Transactions on Computer Systems, Vol. 33, No. 1, Article 2, Publication date: March 2015.

[35] Dabbagh M., Rayes A. , Energy-Efficient Resource Allocation and Provisioning Framework for Cloud Data Centers, IEEE Transactions on Network and Service Management, December 2015.

[36] Farahnakian F. et al., "Using ant colony system to consolidate VMs for green cloud computing," IEEE Trans. Serv. Comput., vol. 8, no. 2, pp. 187198, Apr. 2015.

[37] Xiong P. et al, SmartSLA: Cost-Sensitive Management of 
Virtualized Resources for CPU-Bound Database Services, IEEE Transactions On Parallel And Distributed Systems, VOL. 26, NO. 5, MAY 2015.

[38] Baruchi A. et al, Reducing Virtual Machine Live Migration Overhead via Workload Analysis, IEEE Latin America Transactions, VOL. 13, NO. 4, APRIL 2015.

[39] Tang M., Pan S., A Hybrid Genetic Algorithm for the Energy-efficient Virtual Machine Placement Problem in Data Centers, Neural Processing Letters, springer, 2015.

[40] Alzamil I., Djemame K., Arstrong D., Kavanagh R., Energy-Aware Profiling for Cloud Computing Environments. Electronic Notes in Theoretical Computer Science, Elsevier, 2015.

[41] Chen H., Zhu X., Guo H., Zhu J., Qin X., Wu J., Towards Energy-Efficient Scheduling for Real-time tasks under uncertain Cloud Computing, Journal of System and Software Vol. 99, pp. 20-35, Elsevier, 2015.

[42] Xiao Z., Song W., Chen Q., Dynamic Resource Allocation using Virtual Machines for Cloud Computing Environment. IEEE Transaction on Parallel and Distributed Systems, Vol. 24, No. 6, 2013.

[43] Baloglazov A., Buyya R., Managing Overloaded Hosts for Dynamic Consolidation of Virtual Machines in Cloud Data Centers Under Quality of Service Constraints. IEEE Transactions on Parallel and Distributed System, 2013, pp. 1366-1379.

[44] Beloglazov A., Buyya R., Optimal Online Deterministic Algorithms and Adaptive Heuristics for Energy and Performance Efficient Dynamic Consolidation of Virtual Machines in Cloud Data Centers, CONCURRENCY AND COMPUTATION: PRACTICE AND EXPERIENCE, Wiley
InterScience, 2012.

[45] Wu G., Tang M., Tian Y.C., Li W., Energy-efficient Virtual Machine Placement Problem in Data Centers by Genetic Algorithms, Neural Processing Letters, springer, 2012.

[46] Sego L. H., Marquez A., Rawson A., Cader T., Fox K., Gustafson W. I., Mundy C.J., Implementing the Data Center Energy Productivity Metric. ACM Journal on Emerging Technologies in Computing Systems, 2012.

[47] Anagnostopoulou V., Biswas S., Saadeldeen H., Savage A., Bianchini R, Yang T., Franklin D., and Chong F. T., "Barely alive memory servers: Keeping data active in a low-power state," ACM Journal on Emerging Technologies in Computing Systems [JETC], vol. 8, no. 4, pp. 31:1-31:20, 2012.

[48] Kaplan J.M., Forrest W., Kinder Noah, Revolutionizing Data Center Energy Efficiency, Mckinsey \& Company, july 2008.

[49] Nathuji R., Schwan K., Virtual power: coordinated power management in virtualized enterprise systems, ACM SIGOPS Operating Systems Review 41 [6] , pp. 265-278, 2007.

[50]. Sharma R.K , Bash C.E, Patel C.D, Freidrich R.J, Chase J.S., Balance of power: Dynamic thermal management for internet data centers. IEEE Internet Computing [Volume:9, Issue: 1], pp. 42-49, IEEE, 2005.

[51] Elnozahy E.N.M., Kistler M., Rajamony R., Energy-efficient server clusters. Power-Aware Computer Systems, Springer, 2002.

[52] Dhir V. et. al., Architectural and Qos Issues in Mobile Cloud Computing Environment for Real-Time Video Streaming, International Journal of Advanced Computer Science and Applications, Vol. 7, No. 1, 2016. 